\title{
BENJAMIN OSGOOD PEIRCE
}

Benjamin Osgood Peirce, Hollis professor of mathematics and natural philosophy in Harvard University, Past-president of the American Physical Society and recent member of the editorial board of the PHysical Review, died at Cambridge, Massachusetts, on the fourteenth day of January, I914, in his sixtieth year. 